\title{
Association of antenatal steroids with surfactant administration in moderate preterm infants born to women with diabetes mellitus and/or hypertension
}

\author{
Heather M. Weydig (iD ${ }^{1}$, Charles R. Rosenfeld (iD ${ }^{1}$, Myra H. Wyckoff ${ }^{1}$, Mambarambath A. Jaleel ${ }^{1}$, Patti J. Burchfield ${ }^{1}$, Anita Thomas ${ }^{1}$,
} Mackenzie S. Frost ${ }^{1,2}$ and Luc P. Brion (iD ${ }^{1 凶}$

(c) The Author(s), under exclusive licence to Springer Nature America, Inc. 2021

BACKGROUND: Randomized trials of antenatal steroid administration (ANS) for extreme or moderate preterm pregnancies excluded women with diabetes mellitus (DM) and included few with preeclampsia.

METHODS: Cohort study $(n=1,813)$ including moderate preterm births $\left[29^{0 / 7}-33^{6 / 7}\right.$ wks' gestational age GA)] before (Epoch- 1$)$ and after (Epoch-2) expansion of ANS administration to women with hypertensive disorders (HTN) and/or DM. We compared surfactant administration in Group-1 (neither HTN nor DM), Group-2a (HTN not DM), Group-2b (DM not HTN) and Group-2c (DM and HTN). RESULTS: Surfactant administration was less frequent after ANS in Group-1 [adjusted odds ratio (aOR) $0.54,95 \%$ confidence interval (Cl) $0.31,0.93, P=0.03$ ], Group-2a (aOR 0.36, $\mathrm{Cl} 0.22,0.58, P<0.001$ ) and Group-2c (aOR 0.29, Cl 0.12, 0.71, $P=0.007$ ) but not Group$2 \mathrm{~b}(P=0.64)$.

CONCLUSIONS: ANS administration was independently associated with less surfactant administration in moderately preterm neonates whose mothers had neither HTN nor DM, and those with HTN, but not those with DM without HTN.

Journal of Perinatology (2022) 42:993-1000; https://doi.org/10.1038/s41372-021-01273-6

\section{INTRODUCTION}

Antenatal corticosteroid (ANS) administration reduces the risk of perinatal and neonatal morbidities including mortality, respiratory distress syndrome (RDS), mechanical ventilation and intraventricular hemorrhage (IVH) [1-3].

Based on a lack of evidence, pregnant women with gestational hypertension, preeclampsia or eclampsia (HTN) and/or with diabetes mellitus (DM) were excluded from ANS administration at Parkland Hospital for several years [4,5]. On September 21, 2015, a bundle of care for extremely low gestational age (GA) neonates $\left(23^{0 / 7}-28^{6 / 7}\right.$ weeks) (ELGANs) was introduced [5]. The bundle of care included sequential steps, one of which was expansion of maternal ANS administration to pregnant women with HTN and/or DM and at-risk for delivery [5]. This expansion involved mothers at risk for delivery at $24^{0 / 7}-33^{6 / 7}$ weeks. We have previously shown that maternal ANS administration to women with HTN and/or DM delivering at $23^{0 / 7}-28^{6 / 7}$ weeks GA was associated with an improvement in severe IVH and death or severe IVH but not in surfactant administration [5]. Since the risk of RDS may decrease with maternal HTN but increase with maternal DM [6-12], we aimed in the current report at comparing neonatal outcomes after ANS administration to women with/without HTN and with/without DM at risk for moderate preterm delivery $\left(29^{\circ /}\right.$ ${ }^{7}-33^{6 / 7}$ weeks GA).

Expansion of ANS administration to pregnant women with HTN and/or DM and at-risk for delivery at $29^{0 / 7}-33^{6 / 7}$ weeks GA allowed us to design a unique study in which we could assess: (1) the relationship between the introduction of maternal ANS administration and outcomes in moderate preterm neonates born to women with HTN and/or DM versus those born to women with neither HTN nor DM, (2) the relationship between ANS administration and surfactant administration. Lack of ANS administration to women with HTN and/or DM and at-risk for delivery at $29^{0 / 7}-33^{6 / 7}$ weeks GA at Parkland Hospital allowed us to compare outcomes at Parkland vs other centers participating in the Moderate Preterm Registry from the Eunice Kennedy Shriver National Institute of Child Health and Human Development (NICHD) Neonatal Research Network (NRN), which was a short-term cohort run cohort including moderate preterm neonates delivered from February 1st, 2012 until November 30, $2013[13,14]$.

The primary a-priori hypothesis was that maternal ANS administration would be associated with a lower frequency of surfactant administration in moderate preterm infants including those born to mothers with HTN and/or DM.

\section{METHODS}

Design

This study in moderate preterm neonates included two sections. First, a birth cohort study included retrospective (from April 25, 2011 until September 30, 2014, $n=740$ ) and prospective (from October 1st, 2014 until December 31st, 2018, $n=1073$ ) data, similar to the study in ELGANs recently reported [5]. Second, a subgroup of neonates born at Parkland Hospital between February

${ }^{1}$ Division of Neonatal-Perinatal Medicine, Department of Pediatrics, University of Texas Southwestern Medical Center, Dallas, TX, USA. ${ }^{2}$ Division of Neonatology, Department of

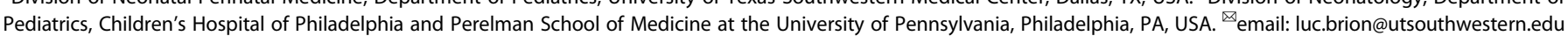

Received: 6 August 2021 Revised: 7 October 2021 Accepted: 5 November 2021

Published online: 20 November 2021 
2012 and November 2013 was compared with those from other centers in the NICHD NRN Moderate Preterm Registry by subtracting data from Parkland Hospital from those for all NRN centers $[13,14]$.

\section{Setting}

Parkland Hospital is a public hospital serving Dallas County Texas with $>12,000$ annual deliveries. All moderate preterm deliveries are attended by a specialized NICU resuscitation team which is comprised of a respiratory therapist, resuscitation nurse, and neonatal nurse practitioner or pediatric resident, with additional help as needed from a NICU fellow and/or neonatology attending. Skills and knowledge of NRP guidelines, including steps to achieve effective ventilation, are regularly assessed and reinforced using simulation and ongoing training. All neonates born at $\leq 32^{6 / 7}$ weeks GA who did not require positive pressure ventilation as per guidelines of the Neonatal Resuscitation Program (NRP) were initially stabilized on face mask continuous positive airway pressure (CPAP) at $5 \mathrm{~cm} \mathrm{H}_{2} \mathrm{O}$ upon delivery using a T-piece resuscitator. If CPAP was required in the delivery room (DR), neonates were transported to the neonatal intensive care unit (NICU) using binasal prong CPAP. Additionally, all neonates in this study were started on $21 \% \mathrm{O}_{2}$ as published [15]. Supplemental $\mathrm{O}_{2}$ was administered if necessary to meet expected values of preductal saturation as recommended by the NRP $[16,17]$. Endotracheal intubation, mechanical ventilation, and surfactant therapy were considered by the care team in the NICU if the neonate had severe respiratory acidosis or high oxygen needs despite CPAP. Surfactant replacement therapy was indicated within one hour after endotracheal intubation for ventilated neonates with radiographic RDS requiring $>40 \% \mathrm{O}_{2}$. There was no policy on surfactant and no change in guidelines on treatment of RDS during the study. Intravenous glucose infusion was started immediately upon admission.

\section{Inclusion/Exclusion}

The birth cohort included inborn preterm neonates delivered at $29^{0 / 7}-33^{6 / 7}$ weeks GA at Parkland Hospital from April 25, 2011 (when routine DR-CPAP and $\mathrm{FiO}_{2}$ starting at 0.21 was implemented for neonates born at $\leq 32^{6 / 7}$ weeks GA [15]) until December 31, 2018 (before implementation of less invasive surfactant administration). GA was based on the best obstetrical estimate. A subset of that birth cohort was compared with neonates from other centers in the NICHD NRN Moderate Preterm Registry, which included moderate preterm neonates delivered from February 1st, 2012 until November 30, 2013 $[13,14]$. Neonates who received comfort care only (without any attempt of resuscitation or any painful procedure) from birth were excluded, except for comparison of ethnicity at Parkland Hospital versus other centers, which was only possible using a reference that did not exclude comfort care [13].

\section{Intervention}

In Epoch-1 (April 25, 2011-September 20, 2015) ANS administration (two doses of $12 \mathrm{mg}$ betamethasone acetate/betamethasone sodium phosphate [Celestone] $24 \mathrm{~h}$ apart) was limited to pregnant women with anticipated delivery at $29^{0 / 7}-33^{6 / 7}$ weeks GA in Group-1, i.e., women with neither DM nor HTN [per American College of Obstetricians and Gynecologists classification]. In Epoch-2 (September 21, 2015-December $31,2018)$ ANS administration was expanded to also include women in Group-2, i.e., those with HTN and/or DM.

\section{Data extraction}

Outcome variables were extracted from two prospectively collected databases: the Neonatal Resuscitation Registry and the NICU Database, which has been validated on several occasions $[18,19]$. Data missing in either database including all those on insulin-dependent DM and any data on primary and secondary outcome variables were collected by review of individual electronic health records. Data from other centers participating in the NICHD NRN Moderate Preterm Registry NRN were calculated as a group by subtracting data from Parkland Hospital from those published for all centers $[13,14]$.

\section{Outcome variables}

The primary outcome was surfactant administration after ANS exposure. This outcome was selected as primary outcome to reflect the clinical severity of lung disease. Other outcomes included DR-ETT, germinal matrix hemorrhage or IVH (Papile's classification) [20] (GMH-IVH), and in-hospital mortality. Since surfactant administration is a subjective variable, we added $\mathrm{RDS}$ (defined as grunting respirations, retractions, and $\mathrm{FiO}_{2}>0.40$ with compatible clinical, radiologic, laboratory, and, if available, autopsy findings), as another outcome post-hoc variable.

\section{Statistical analysis and sample size}

Statistical analyses were done with SPSS version 27 (IBM, Inc, Armonk, NY). Data are presented as means \pm SD (if normally distributed) or number with the percent of the population.

Continuous variables were compared between groups and epochs using two-way analysis of variance followed by Student $t$-tests (or Welch tests) with Bonferroni correction. Nominal variables were compared by general log-linear analysis if $P$ values for group and epoch could be estimated, or else by chi-square $\left(x^{2}\right)$ analysis with exact test, followed by $x^{2}$ analysis for pair-wise comparisons with Bonferroni correction. Parkland data were compared with those in other NRN centers using Student t-test or $x^{2}$ analysis.

Two logistic regression models were used to determine the adjusted odds ratio (aOR) and 95\% confidence interval (Cl) for DR-ETT, need for surfactant administration, RDS, GMH-IVH, or mortality. In the first model, independent association of each outcome variable was assessed based on three variables: Group [Group-2 (maternal DM or HTN) vs. Group-1 (no maternal DM, no HTN)], ANS exposure (ANS-exposed vs. ANS-unexposed neonates), and Epoch [Epoch-2 (after ANS expansion) vs. Epoch-1 (before ANS expansion)]. In addition, Group-2 neonates (maternal HTN and/or DM) were compared with Group-1 neonates (no maternal DM, no HTN) in the absence of ANS.

In the second model, a forward stepwise logistic regression was used to adjust the OR of each outcome variable for candidate variables available at birth. The latter included Epoch, Group, ANS, race and ethnicity (classified based on maternal records as Hispanic, Black not Hispanic, or other), chorioamnionitis, premature rupture of membranes, GA, birthweight Z-score (calculated using Olsen's data) [21], sex, one-min Apgar score less than 5 , and (for mortality) congenital malformations. For these multivariate analyses, birthweight Z-scores were used rather than a dichotomous variable such as small for GA (SGA) or fetal growth restriction because in a previous study at Parkland Hospital neonatal morbidity and mortality progressively decreased with increasing weight centiles [22]. Subgroup analyses were done using forward stepwise logistic regression models to assess whether the likelihood of the outcome was independently associated with ANS exposure among all neonates included in Group-1 (neither HTN nor DM), Group-2 (HTN and/or DM), Group-2a (HTN, not DM), Group-2b (DM, not HTN) and Group-2c (DM and HTN).

Multiple secondary analyses were not adjusted for multiple testing and did not include sample size analyses; therefore, these analyses should be considered exploratory.

A total sample size of 450 was calculated using SAS 9.4 (SAS Institute, Inc, Cary, NC) to have $80 \%$ power with a two-tailed alpha of 0.05 to detect an aOR of 0.5 for surfactant administration assuming 30\% estimate without ANS using logistic regression adjusted for GA and sex with alpha error $<0.05$. Logistic regression models were limited to one variable per ten subjects with the outcome of interest [23].

\section{RESULTS \\ Demographics}

Among 1,824 moderate preterm neonates, five neonates in Epoch-1 and six in Epoch-2 received comfort care only and were excluded from further analyses. Among the remaining 1,813 moderate preterm neonates, 950 were in Epoch-1 and 863 in Epoch-2 (Fig. 1). They were further classified as described earlier as neonates born to women: with neither HTN nor DM (Group-1), with HTN but without DM (Group-2a), DM but without HTN (Group-2b), and both DM and HTN (Group-2c). In Epoch-2 the racial/ethnic distribution changed with more White not Hispanic and fewer Hispanic than in Epoch-1 (Table 1). Neonates in Group-2 had a lower average birthweight $(P<0.001)$ and were more frequently SGA $(P<0.001)$ than those in Group-1, as expected from the high proportion of mothers with HTN in Group-2.

\section{Intervention: introduction of ANS administration to women with HTN and/or DM}

Neonates in Group-1, Epoch-1 had greater ANS exposure than those in Group-2, consistent with the clinical practice at that time 


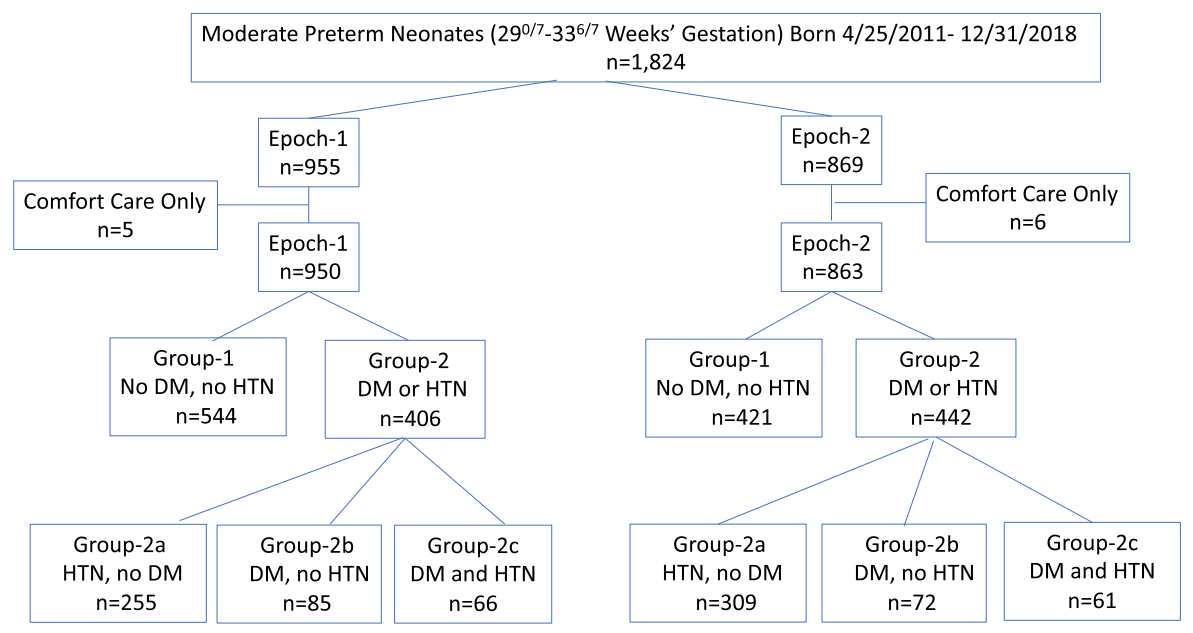

Fig. 1 Flow diagram. Abbreviations: DM, diabetes mellitus; HTN, gestational hypertension, preeclampsia or eclampsia.

Table 1. Comparison of maternal and neonatal clinical characteristics in Epoch-1 and Epoch-2 by Group.

\begin{tabular}{|c|c|c|c|c|c|c|}
\hline \multirow[t]{2}{*}{ Characteristic* } & \multicolumn{2}{|c|}{$\begin{array}{l}\text { Epoch-1 April 25, 2011- September 20, } 2015 \\
n=950\end{array}$} & \multicolumn{2}{|c|}{$\begin{array}{l}\text { Epoch-2 September 21, } 2015 \text { - December } \\
2018 n=863\end{array}$} & \multicolumn{2}{|c|}{$P$ value** } \\
\hline & $\begin{array}{l}\text { Group-1 No } \\
\text { maternal HTN/DM } \\
(n=544)\end{array}$ & $\begin{array}{l}\text { Group-2 Maternal } \\
\text { HTN or DM }(n= \\
406)\end{array}$ & $\begin{array}{l}\text { Group-1 No } \\
\text { maternal HTN/DM } \\
(n=421)\end{array}$ & $\begin{array}{l}\text { Group-2 Maternal } \\
\text { HTN or DM }(n= \\
442)\end{array}$ & Epoch & Group \\
\hline Maternal diabetes mellitus\# & $0^{\mathrm{a}}$ & $151(37 \%)^{b}$ & $0^{\mathrm{a}}$ & $133(30 \%)^{b}$ & 0.78 & $<0.001$ \\
\hline HTN\# & $0^{a}$ & $321(79 \%)^{b}$ & $0^{\mathrm{a}}$ & $370(84 \%)^{b}$ & 0.06 & $<0.001$ \\
\hline $\begin{array}{l}\text { Premature prolonged } \\
\text { rupture of membranes }\end{array}$ & $203(37 \%)^{\mathrm{a}}$ & $55(14 \%)^{b}$ & $157(37 \%)^{a}$ & $54(12 \%)^{b}$ & 0.03 & $<0.001$ \\
\hline Birth weight (g) & $1820 \pm 403^{a}$ & $1659 \pm 442^{b}$ & $1828 \pm 396^{\mathrm{a}}$ & $1717 \pm 410^{b}$ & 0.10 & $<0.001$ \\
\hline Gestational age (weeks) & $31.5 \pm 1.4$ & $31.6 \pm 1.3$ & $31.7 \pm 1.3$ & $31.5 \pm 1.4$ & 0.74 & 0.35 \\
\hline Small for gestational age & $28(5 \%)^{a}$ & $71(18 \%)^{b}$ & $27(6 \%)^{a}$ & $46(10 \%)^{c}$ & 0.048 & $<0.001$ \\
\hline Black not Hispanic & $110(20 \%)$ & $90(22 \%)$ & $93(22 \%)$ & $122(28 \%)$ & & \\
\hline White not Hispanic & $28(5 \%)^{a}$ & $16(4 \%)$ & $40(10 \%)^{b}$ & $20(5 \%)$ & & \\
\hline Other & $21(4 \%)$ & $9(2 \%)$ & $8(2 \%)$ & $8(2 \%)$ & & \\
\hline 1-minute Apgar score $<5$ & $141(26 \%)$ & $107(27 \%)$ & $96(23 \%)$ & $112(25 \%)$ & 0.06 & 0.40 \\
\hline 5-minute Apgar score $<5$ & $35(6 \%)^{a}$ & $18(4 \%)$ & $13(3 \%)^{b}$ & $14(3 \%)$ & 0.004 & 0.08 \\
\hline $\begin{array}{l}\text { HTN includes gestational hyp } \\
{ }^{*} \text { Values are mean } \pm \text { SD or nun } \\
{ }^{* *} \text { Chi-square (diabetes mellitu } \\
\text { ANOVA followed by Student } \\
\text { a,b Different superscripts acros } \\
{ }^{* * *} \text { interaction between Epoch }\end{array}$ & $\begin{array}{l}\text { ansion, preeclampsia o } \\
\text { ar (\%). } \\
\text { st HTN) or general log } \\
\text { st for continuous varia } \\
\text { row indicate significa } \\
\text { d Group, } P=0.001\end{array}$ & $\begin{array}{l}\text { lampsia, } D M \text { diabetes me } \\
\text { ar analysis (other variable }\end{array}$ & $\begin{array}{l}\text { itus. } \\
\text { followed by chi }\end{array}$ & (\#exact test) for $r$ & . & vo-way \\
\hline
\end{tabular}

(Table 1). ANS exposure increased significantly to $88 \%$ in Group-1 and $92 \%$ in Group-2 during Epoch-2 (Table 1).

\section{DR-ETT}

In the absence of ANS, $12-20 \%$ of moderate preterm neonates were intubated in the DR in Groups-1 and -2 and within each Epoch (Table 2). In the first logistic regression model, DR-ETT was less frequent in Epoch-2 than in Epoch-1 and less frequent after ANS exposure, but not significantly different between Groups-1 and -2 (Table 2).

In the stepwise logistic regression model, the likelihood of DR-ETT decreased in Epoch-2 (aOR 0.47, Cl 0.33, 0.66, $P<0.001$ ), decreased with increasing GA (aOR 0.71, Cl 0.62, 0.80, $P<0.001$ ) and was higher in neonates with a one-minute Apgar score $<5$ (aOR 10.4, Cl 7.3, 14.8, $P<0.001$ ). The likelihood of DR-ETT was not affected by ANS exposure, group, sex, birthweight Z-score, chorioamnionitis, and premature prolonged rupture of membranes.

\section{Surfactant administration and RDS}

In the absence of ANS, 14-29\% of moderate preterm neonates received surfactant therapy in Epochs- 1 and -2 and Groups- 1 and -2 (Table 2). In the first logistic regression model, surfactant 
Table 2. Comparisons of morbidities and mortality in neonates by Epoch, Group and ANS exposure.

\begin{tabular}{|c|c|c|c|c|c|c|c|c|}
\hline \multirow[t]{2}{*}{ Outcome } & \multirow[t]{2}{*}{ ANS } & \multicolumn{2}{|c|}{$\begin{array}{l}\text { Epoch-1 April 25, 2011- September 20, } \\
2015 n=950\end{array}$} & \multicolumn{2}{|c|}{$\begin{array}{l}\text { Epoch-2 September 21, } 2015 \text { - December } \\
2018 n=863\end{array}$} & \multicolumn{3}{|l|}{$P$ value } \\
\hline & & $\begin{array}{l}\text { Group-1 No } \\
\text { maternal HTN/DM }\end{array}$ & $\begin{array}{l}\text { Group-2 } \\
\text { Maternal } \\
\text { HTN or DM }\end{array}$ & $\begin{array}{l}\text { Group-1 No } \\
\text { maternal HTN/DM }\end{array}$ & $\begin{array}{l}\text { Group-2 } \\
\text { Maternal } \\
\text { HTN or DM }\end{array}$ & Epoch & Group & ANS \\
\hline \multirow{2}{*}{$\begin{array}{l}\text { ETT in } \\
\text { delivery room }\end{array}$} & No & 19/105 (18\%) & $41 / 349(12 \%)$ & $9 / 52(17 \%)^{a}$ & $7 / 35(20 \%)^{a}$ & \multirow[t]{2}{*}{0.002} & \multirow[t]{2}{*}{0.050} & \multirow[t]{2}{*}{0.047} \\
\hline & Yes & $62 / 439(14 \%)$ & 9/57 (16\%) & $24 / 369(7 \%)^{b}$ & $24 / 407(6 \%)^{b}$ & & & \\
\hline $\begin{array}{l}\text { Surfactant } \\
\text { administration }\end{array}$ & No & $25 / 105(24 \%)^{a}$ & $101 / 349(29 \%)^{a}$ & $7 / 52(14 \%)$ & $10 / 35(29 \%)$ & 0.32 & 0.002 & $<0.001$ \\
\hline \multirow[t]{2}{*}{$\mathrm{GMH}^{-I V H}{ }^{*}$} & No & 11/71 (16\%) & $60 / 231(26 \%)$ & $6 / 27(22 \%)$ & $6 / 26(23 \%)$ & \multirow[t]{2}{*}{0.59} & \multirow[t]{2}{*}{0.12} & \multirow[t]{2}{*}{0.20} \\
\hline & Yes & 49/262 (19\%) & $3 / 38(8 \%)$ & $26 / 192(14 \%)$ & $49 / 246(20 \%)$ & & & \\
\hline \multirow[t]{2}{*}{ Mortality } & No & $2 / 105(2 \%)$ & $2 / 349(1 \%)$ & $3 / 52(6 \%)$ & $2 / 35(6 \%)$ & \multirow[t]{2}{*}{0.25} & \multirow[t]{2}{*}{0.79} & \multirow[t]{2}{*}{0.58} \\
\hline & Yes & $7 / 439(2 \%)$ & $2 / 57(3 \%)$ & $4 / 369(1 \%)$ & $8 / 407$ (2\%) & & & \\
\hline
\end{tabular}

Logistic regression analysis including three variables: Epoch, ANS, and Group.

In this model, ANS exposure was associated with less ETT in the delivery room and less surfactant administration but not with GMH-IVH or mortality. Different superscripts down a column indicate significant pair-wise differences (ANS Yes vs. No) with Bonferroni correction.

$E T T$ endotracheal tube insertion, GMH-IVH germinal matrix hemorrhage or intraventricular hemorrhage, HTN gestational hypertension, preeclampsia or eclampsia, $D M$ diabetes mellitus.

${ }^{*}$ Neurosonogram was obtained in $63.4 \%$ and $56.9 \%$ of neonates in Epoch-1 and Epoch-2, respectively $(P=0.005)$.

administration was higher in Group-2 than Group-1 and lower in ANS-exposed neonates than ANS-unexposed neonates (42\% lower in Group-1 and 44\% in Group-2), but not significantly different between the two epochs (Table 2). Post-hoc analysis showed that the frequency of RDS was higher in Group-2 than Group-1 and lower in ANS-exposed neonates than ANS-unexposed neonates, but not significantly different between the two epochs (data not shown).

In the stepwise logistic regression model (Table 3.a), the aOR of surfactant administration was lower in neonates exposed to ANS $(0.47, \mathrm{Cl} 0.34-0.65, P<0.001)$ than unexposed neonates. The aOR of surfactant administration was also lower in those with premature prolonged rupture of membranes $(P=0.007)$, decreased with increasing GA $(P<0.001)$ and was higher in Group-2 $(P<0.001)$, neonates with 1-minute Apgar score $<5(P=$ $0.01)$ and those who underwent DR-ETT $(P<0.001)$. Surfactant administration was not independently associated with Epoch, birthweight Z-score, race/ethnicity, and sex.

Subgroup analyses showed no independent association of surfactant administration with Epoch (Table 3.b-e). Stepwise logistic regression in all neonates included in Group-1 (neither DM, nor HTN) showed that the aOR of surfactant administration was lower after ANS exposure (aOR 0.54, $\mathrm{Cl} 0.31,0.93, P=0.03$ ), decreased with increasing GA $(P<0.001)$ and was higher in neonates with low five-minute Apgar score $(P=0.04)$ and those who underwent DR-ETT $(P<0.001)$ (Table 3.b). Subgroup analysis in all neonates in Group-2 (HTN and/or DM) showed that the aOR of surfactant administration was lower after ANS exposure (aOR $0.41, \mathrm{Cl} 0.28,0.61, P<0.001)$, decreased with increasing GA $(P<$ $0.001)$ and was higher after prolonged rupture of membranes $(P$ $=0.002)$ and in neonates who DR-ETT $(P<0.001)$ (Table 3.c). Subgroup analysis in all neonates in Group-2a (HTN, but not DM) showed that the aOR of surfactant administration was lower with ANS exposure (aOR 0.36, $\mathrm{Cl} 0.22,0.58, P<0.001)$, decreased with increasing GA $(P<0.001)$ and was higher in neonates who had DRETT $(P<0.001)$ (Table 3.d). In contrast, subgroup analysis in Group2b (maternal DM, not HTN) suggested that surfactant administration was not independently associated with ANS exposure $(P=$ $0.67)$ but decreased with increasing GA $(P<0.001)$ and was higher in neonates who had DR-ETT $(P<0.001)$ (Table 3.e). However, the sample size in Group-2b was insufficient to rule out an association with ANS. In Group-2c (DM and HTN), surfactant administration was independently associated with ANS exposure (aOR $0.29, \mathrm{Cl}$
0.12-0.71, $P=0.007)$, decreased with increasing GA $(P<0.001)$ and was higher in neonates who had DR-ETT ( $P=0.04)$ (Table 3.f). Post-hoc multivariate analysis showed that the frequency of RDS was lower after ANS administration in neonates born to women with HTN $(P=0.006)$ but not in those born to women with DM but not HTN $(P=0.995)$; the frequency decreased with increasing GA, but did not change with Epoch (data not shown).

In summary, surfactant administration and RDS decreased with increasing GA, but was not independently associated with Epoch. Surfactant administration and RDS were independently associated with ANS exposure in mothers with HTN. However, the effect of ANS in moderate preterm neonates delivered of women with DM but not HTN is unclear.

\section{Mortality}

In the absence of ANS, in-hospital mortality was not significantly different in Group-1 vs Group-2 (3.2\% vs. $1.0 \%$, respectively, $P=$ 0.08). In the first logistic regression model, mortality was unaffected by ANS, Epoch, and Group (Table 2). In the stepwise logistic regression model, mortality was associated with congenital anomaly (aOR 12.00,95\% Cl 5.75, 25.06, $P<0.001)$, but unaffected by GA, ANS, Epoch, race, sex, and Group.

\section{GMH-IVH}

Neurosonogram was obtained more frequently in Epoch-1 than Epoch-2 (63.4\% vs 56.9\%, respectively, $P=0.005)$. In the absence of ANS the frequency of GMH-IVH was not significantly different between Group-1 and Group-2 (Table 2). In the first logistic regression model, the frequency of GMH-IVH was unaffected by ANS, Group, and Epoch (Table 2).

In stepwise forward logistic regression analysis $(n=1,093)$, the frequency of GMH-IVH decreased with ANS exposure (aOR 0.67, $95 \% \mathrm{Cl} 0.49,0.92, P=0.01$ ), increasing $\mathrm{GA}$ (aOR $0.77,95 \% \mathrm{Cl} 0.68$, $0.87, P<0.001)$, increasing birthweight Z-score (aOR $0.87,95 \% \mathrm{Cl}$ $0.75,0.995, P=0.04)$ and was higher in males (aOR $1.43,95 \% \mathrm{Cl}$ $1.05,1.95, P=0.02)$. Subgroup analysis in all neonates in Group-1 (neither HTN nor DM, $n=552$ ) showed that the frequency of GMH-IVH decreased with increasing GA (aOR 0.78, Cl 0.65, 0.94, $P$ $=0.009$ ) and was higher after premature rupture of membranes (aOR 1.84, Cl 1.15, 2.95, $P=0.01$ ) and DR-ETT (aOR 2.38, Cl 1.40, $4.05, P=0.001)$, but was not related to ANS exposure. Subgroup analysis in all neonates in Group-2 (HTN and/or DM, $n=541$ ) showed that the aOR of GMH-IVH was lower with ANS exposure 
Table 3. Multivariate analyses of variables affecting the odds ratio of surfactant administration in various groups. 3.a All 29-33 weeks' GA neonates (surfactant administration in 313 among 1813 neonates). 3.b Group-1: No maternal HTN/DM (surfactant administration in 127 among 965 neonates). 3.c Group-2: Maternal HTN or DM (surfactant administration in 186 among 848 neonates). 3.d Group-2a: Maternal HTN, no DM (surfactant administration in 119 among 564 neonates). e Group-2b: Maternal DM, no HTN (surfactant administration in 26 among 157 neonates)*. $3 . f$ Group-2c: Maternal HTN and DM (surfactant administration in 41 among 127 neonates).

\section{3.a. Variable}

Antenatal steroid administration

Group-2 vs. Group-1

Premature prolonged rupture of membranes

Gestational age (per week)

1-minute Apgar score $<5$

Endotracheal intubation in the delivery room

\section{3.b. Variable}

Antenatal steroid administration

Gestational age (per week)

1-minute Apgar score $<5$

Endotracheal intubation in the delivery room

3.c. Variable

Antenatal steroid administration

Premature prolonged rupture of membranes

Gestational age (per week)

Endotracheal intubation in the delivery room

\section{3.d. Variable}

Antenatal steroid administration

Gestational age (per week)

Endotracheal intubation in the delivery room

\section{3.e. Variable}

Gestational age (per week)

Endotracheal intubation in the delivery room

\section{3.f. Variable}

Antenatal steroid administration

Gestational age (per week)

Endotracheal intubation in the delivery room

\begin{tabular}{|c|c|}
\hline Adjusted odds ratio ( $95 \%$ confidence interval) & $P$ Value \\
\hline $0.47(0.34,0.65)$ & $<0.001$ \\
\hline $1.82(1.33,2.49)$ & $<0.001$ \\
\hline $0.58(0.38,0.86)$ & 0.007 \\
\hline $0.48(0.43,0.53)$ & $<0.001$ \\
\hline $1.52(1.10,2.11)$ & 0.01 \\
\hline $7.43(4.96,11.13)$ & $<0.001$ \\
\hline Adjusted odds ratio ( $95 \%$ confidence interval) & $P$ Value \\
\hline $0.54(0.31,0.93)$ & 0.03 \\
\hline $0.51(0.43,0.60)$ & $<0.001$ \\
\hline $1.67(1.02,2.74)$ & 0.04 \\
\hline $9.41(5.52,16.04)$ & $<0.001$ \\
\hline Adjusted odds ratio ( $95 \%$ confidence interval) & $P$ Value \\
\hline $0.41(0.28,0.61)$ & $<0.001$ \\
\hline $0.31(0.15,0.65)$ & 0.002 \\
\hline $0.45(0.39,0.52)$ & $<0.001$ \\
\hline $7.12(4.00,12.80)$ & $<0.001$ \\
\hline Adjusted odds ratio ( $95 \%$ confidence interval) & $P$ Value \\
\hline $0.36(0.22,0.58)$ & $<0.001$ \\
\hline $0.41(0.33,0.49)$ & $<0.001$ \\
\hline $6.97(3.09,15.72)$ & $<0.001$ \\
\hline Adjusted odds ratio ( $95 \%$ confidence interval) & $P$ Value \\
\hline $0.42(0.29,0.61)$ & $<0.001$ \\
\hline $12.02(3.68,39.33)$ & $<0.001$ \\
\hline Adjusted odds ratio ( $95 \%$ confidence interval) & $P$ Value \\
\hline $0.29(0.12,0.71)$ & 0.007 \\
\hline $0.56(0.40,0.78)$ & $<0.001$ \\
\hline $3.79(1.05,13.73)$ & 0.04 \\
\hline
\end{tabular}

Stepwise logistic regression analyses using the following potential variables: Epoch, Group (Table 3.a only), ANS, race and ethnicity, chorioamnionitis, premature rupture of membranes, GA, birthweight Z-score, sex, 1-min Apgar score less than 5.

ANS antenatal steroid, HTN gestational hypertension, preeclampsia or eclampsia, DM diabetes mellitus.

alimited to two variables because $<30$ neonates received surfactant.

(aOR 0.60, $\mathrm{Cl} 0.40,0.91, P=0.02$ ), decreased with increasing GA $(0.84, \mathrm{Cl} 0.72,0.98, P=0.03)$ and was higher after chorioamnionitis (aOR $4.62, \mathrm{Cl} 1.20,17.82, P=0.03$ ). The frequency of severe IVH was insufficient for multivariate analyses and subgroup analyses.

\section{Comparison of Parkland subgroup with the Moderate Preterm Registry from the NICHD NRN}

Between February 2012 and November 2013, 386 moderate preterm neonates (383 excluding comfort care only) were delivered at Parkland Hospital and 6,671 (6,631 excluding comfort care only) in other centers participating in the moderate preterm registry of the NICHD NRN (Table 4). Neonates born at Parkland Hospital had similar average GA but greater average birthweight than those born in other centers and were less likely to be SGA. Despite a lower frequency of ANS exposure at Parkland Hospital $(P<0.001)$ than in other centers, the frequency of DR-ETT and that of surfactant administration at Parkland Hospital were significantly lower $(P=0.005)$. Two thirds of neonates who were administered surfactant had undergone DR-ETT at Parkland and in other centers. The frequency of DR-ETT without cardiopulmonary resuscitation $(\mathrm{CPR})$ at Parkland Hospital did not differ from other centers; however, the frequency of DR-CPR and that of DR-ETT and/or DR-CPR were lower at Parkland than in other centers $(P<$ 0.001 and $P=0.02$, respectively).

\section{DISCUSSION}

Administration of ANS to women with HTN and/or DM in our institution was limited for several years since women with DM had been excluded from existing randomized controlled trials (RCTs). There was also a clinical concern for delaying timely delivery in high-risk pregnancies with HTN, in order to administer a course of ANS, might result in increased fetal demise and/or maternal morbidity. Subsequent expansion of ANS administration to women with HTN and/or DM uniquely allowed us to examine the hypothesis, i.e., is ANS exposure in moderate preterm neonates associated with decreased surfactant administration for RDS. Our data suggest that the association of ANS exposure with decreased frequency of surfactant administration is similar in moderate preterm neonates born to women with HTN (with or without DM) when compared with those with neither HTN nor DM. There was no association between ANS administration and lower occurrence of DR-ETT. Thus, we provide new evidence supporting efficacy of maternal ANS administration on the 
Table 4. Comparison of Parkland Hospital with the Moderate Preterm Registry from the National Institute of Child Health and Human Development Neonatal Research Network, February 2012 to November 2013.

\begin{tabular}{|c|c|c|c|}
\hline Entire registry* & $\begin{array}{l}\text { Parkland hospital } \\
n=386\end{array}$ & $\begin{array}{l}\text { Other NRN centers } \\
n=6,671\end{array}$ & $P$ Value \\
\hline Excluding neonates who received comfort care only** & $n=383$ & $n=6,631$ & \\
\hline Insulin-dependent diabetes. mellitus & $25 / 383(6.5 \%)$ & $498 / 6,612(7.5 \%)$ & 0.47 \\
\hline Chorioamnionitis & $11 / 383(2.9 \%)$ & $484 / 6,561(7.4 \%)$ & $<0.001$ \\
\hline Gestational age (weeks) & $31.5 \pm 1.4$ & $31.5 \pm 1.4$ & $>0.99$ \\
\hline Birth weight (grams) & $1,747 \pm 427$ & $1,693 \pm 429$ & 0.02 \\
\hline Small for gestational age & $33 / 383(8.6 \%)$ & $1118 / 6,626(16.9 \%)$ & $<0.001$ \\
\hline 5 -minute Apgar score $<5$ & $20 / 383(5.2 \%)$ & $322 / 6,586(4.9 \%)$ & 0.77 \\
\hline Endotracheal intubation in the delivery room without CPR & $49 / 383(12.8 \%)$ & $984 / 6,631(14.8 \%)$ & 0.27 \\
\hline Endotracheal intubation in the delivery room among neonates who received surfactant & $50 / 72(69.4 \%)$ & $1170 / 1,753(66.7 \%)$ & 0.63 \\
\hline $\begin{array}{l}\text { Values are mean } \pm \mathrm{SD} \text { or number/total }(\%) \text {. } \\
\text { Student } t \text {-test, chi-square analysis or Fisher's exact test } \\
\text { Abbreviations: HTN, gestational hypertension, preeclampsia or eclampsia; CPR, cardiopulmor } \\
\text { aData from reference [6] } \\
\text { bdata from reference [7] }\end{array}$ & reit & - & \\
\hline
\end{tabular}

frequency of RDS requiring surfactant therapy in moderate preterm neonates delivered of pregnant women with HTN. Notably, the effect of ANS administration on surfactant administration in moderate preterm neonates delivered of women with DM but no HTN remains unclear.

Maternal DM is associated with an increased risk of neonatal RDS [6]. Phospholipid profile analyses of amniotic fluid have shown that RDS in IDMs is associated with delayed production of phosphatidyl-glycerol and increase in phosphatidyl-inositol $[7,8]$. Several additional mechanisms are involved in IDM-induced RDS, including decreased production of hydrophobic surfactant proteins $B$ and $C$ and inactivation of glycogen phosphorylase A [6, 9-11]. In contrast, pre-eclampsia is associated with high transplacental transport of maternal cortisol due to reduced activity of placental 11 beta-hydroxysteroid dehydrogenase type 2 [12]; this could lead to less RDS and surfactant administration.

The association between ANS exposure and outcomes among preterm neonates born to women with HTN and/or DM appears to differ when GA is considered. In our previous study, we examined ELGANs and observed an independent association of ANS exposure with less frequent severe IVH and death or severe IVH, but not mortality or surfactant administration in neonates born to women with HTN and/or DM [5]. In the current study, we examined moderate preterm neonates, i.e., $29^{0 / 7}-33^{6 / 7}$ weeks GA, and observed an independent association of ANS exposure with decreased surfactant administration and decreased $\mathrm{GMH}-\mathrm{IVH}$, but not DR-ETT or mortality in neonates born to women with HTN. Neonatal mortality was independently associated with congenital anomalies. ANS exposure was independently associated with less surfactant administration in Group-1, Group-2a (HTN without DM) and Group-2c (HTN and DM) but not in Group-2b (DM without HTN). Meta-analysis of RCTs assessing the effects of ANS in a total of 418 women with hypertensive syndromes showed similar effects on multiple outcomes (i.e., IVH, neonatal death, RDS) in comparison with trials in women without HTN [1]. It is notable that the effects of ANS on neonatal outcomes in pregnant women with
DM have not been studied in RCTs and thus the effects remain unclear [4, 24]. In a large US cohort from the NICHD Maternal-Fetal Medicine Network, the authors reported similar neonatal outcomes in association with ANS exposure in women with and those without DM; however, they did not include the diagnosis of RDS or surfactant administration [25].

Notably, we observed that the frequency of DR-ETT and that of surfactant administration before ANS expansion were lower at Parkland Hospital than in other centers within the NICHD NRN despite lower ANS exposure. This difference may be in part related to center heterogeneity in patients and/or practice (e.g., resuscitation team, criteria for DR-ETT and for surfactant administration). The frequency of DR-ETT without CPR was similar at Parkland Hospital and other centers, however, the frequency of DR-ETT with or without CPR was significantly lower at Parkland Hospital. Most intubated neonates at Parkland Hospital did not receive CPR, and CPR was less frequent at Parkland Hospital than in other centers. We speculate that the ongoing training and experience of the resuscitation team in achieving effective ventilation at Parkland Hospital may have been critical.

In the stepwise logistic regression models in this study, the frequency of DR-ETT at Parkland Hospital was not associated with ANS exposure but decreased in Epoch-2, whereas the frequency of surfactant administration was associated with ANS exposure but was unchanged in Epoch-2. Previous studies have shown changes in DR clinical practice at Parkland Hospital associated with: (1) a decrease in DR-ETT in 28-34 weeks GA neonates from 19\% in 2004-2005 to 9\% in 2009-2010 after participation in the SUPPORT trial targeting $24^{0 / 7}-27^{6 /}$ ${ }^{7}$ weeks GA neonates;[26] (2) an increase in DR-CPAP in 35-42 weeks GA neonates after implementation of new NRP guidelines for late preterm and term neonates in 2011;[27] and (3) a quality improvement project implemented in October 2015 to decrease DR-ETT in preterm neonates $\leq 29$ weeks GA, i.e., at almost the same time as expansion of ANS to women with HTN and/or DM [28].

The present study has several strengths. First, perinatal management at Parkland Hospital follows clearly defined guidelines 
established by the Divisions of Maternal-Fetal Medicine and Neonatal-Perinatal Medicine. Second, the cohort was limited to a period without any additional change in relevant perinatal practice in moderate preterm neonates at Parkland Hospital. Specifically, the cohort started after implementation of DR-CPAP for preterm neonates $\leq 32^{6 / 7}$ weeks' GA and initiation of DR management with $21 \% \mathrm{O}_{2}$ in preterm neonates [8], and stopped before initiation of less invasive surfactant administration. Third, data were collected from two prospective registries, including a long standing, well validated NICU database [10, 11], with additional data extraction from the electronic health records. Fourth, sample size was sufficient for examining the primary outcome.

Limitations of the study include (1) lack of randomization of ANS therapy thereby allowing analysis of associations only and preventing any conclusion about causality; (2) multiple secondary analyses with small sample size and without $P$-value adjustment, which should be considered as hypothesis generating, (3) insufficient sample size to assess changes in surfactant administration with ANS in Group-2b (DM without HTN) and multivariate analyses and subgroup analyses of severe IVH; and (4) limitations of data sets included in the NICU database and the resuscitation databases and lack of chart review in all women and neonates for several other potential variables. Therefore we missed the following variables for this study: (1) timing and completeness of ANS administration, which may have been incomplete in Groups-2a and $-2 c$ because of the obstetric concern for an increase in risk in women with severe preeclampsia that may be associated with delaying delivery in order to facilitate fetal maturation; [4, 5] (2) pathologic chorioamnionitis; (3) an objective parameter to assess severity of lung disease, such as ventilator index or oxygen saturation index; $[29,30]$ and (4) hypoglycemia, which has been observed more frequently with increasing GA and is a common complication of ANS in late preterm but not moderate preterm infants [31-33].

In summary, maternal ANS administration at Parkland Hospital is independently associated with decreased frequency of postnatal surfactant administration in moderate preterm neonates delivered from women with HTN (with or without DM) and in those delivered from women with neither HTN nor DM. In our previous study in ELGANs, maternal ANS administration was associated with an improvement in severe IVH and death or severe IVH but not in surfactant administration [5]. Additional studies of the efficacy of ANS in neonates delivered from women with DM are still needed to determine benefit.

\section{DATA AVAILABILITY}

The datasets generated and/or analyzed during the current study are available from the corresponding author on reasonable request.

\section{REFERENCES}

1. McGoldrick E, Stewart F, Parker R, Dalziel SR. Antenatal corticosteroids for accelerating fetal lung maturation for women at risk of preterm birth. Cochrane Database of Syst Rev. 2020;12:CD004454.

2. WHO ACTION Trials Collaborators. The World Health Organization ACTION-I (Antenatal Corticosteroids for Improving Outcomes in preterm Newborns) Trial: a multi-country, multi-centre, two-arm, parallel, double-blind, placebo-controlled, individually randomized trial of antenatal corticosteroids for women at risk of imminent birth in the early preterm period in hospitals in low-resource countries. Trials 2019;20:507.

3. Schmitz T, Alberti C, Ursino M, Baud O, Aupiais C, BETADOSE study group and the GROG (Groupe de Recherche en Gynécologie Obstétrique). Full versus half dose of antenatal betamethasone to prevent severe neonatal respiratory distress syndrome associated with preterm birth: study protocol for a randomised, multicenter, double blind, placebo-controlled, non-inferiority trial (BETADOSE). BMC Pregnancy Childbirth. 2019;19:67.

4. Bloom SL, Leveno KJ. Corticosteroid use in special circumstances: preterm ruptured membranes, hypertension, fetal growth restriction, multiple fetuses. Clin Obstetr Gynecol. 2003;46:150-60.
5. Weydig HM, Rosenfeld CR, Jaleel MA, Burchfield PJ, Frost MS, Brion LP. Association of antenatal steroids and neonatal mortality and morbidity in preterm infants born to mothers with diabetes mellitus and gestational hypertension. J Perinatol. 2021;41:1660-8.

6. Atar YH, Baatz JE, Ryan RM. Molecular mechanisms of maternal diabetes effects on fetal and neonatal surfactant. Child (Basel). 2021;8:281.

7. Moore TR. A comparison of amniotic fluid fetal pulmonary phospholipids in normal and diabetic pregnancy. Am J Obstet Gynecol. 2002;186:641-50.

8. Hallman M, Teramo K. Amniotic fluid phospholipid profile as predictor of fetal maturity in diabetic pregnancies. Obstet Gynecol 1979;54:703-7.

9. Guttentag SH, Phelps DS, Warshaw JB, Floros J. Delayed hydrophobic surfactant protein (SP-B, SP-C) expression in fetuses of streptozotocin-treated rats. Am J Respir Cell Mol Biol. 1992;7:190-7.

10. Rayani HH, Gewolb IH, Floros J. Glucose decreases steady state mRNA content of hydrophobic surfactant proteins $B$ and $C$ in fetal rat lung explants. Exp Lung Res. 1999;25:69-79.

11. Gewolb IH, Barrett C, Wilson CM, Warshaw JB. Delay in pulmonary glycogen degradation in fetuses of streptozotocin diabetic rats. Pediatr Res. 1982;16:869-73.

12. Aufdenblatten M, Baumann M, Raio L, Dick B, Frey BM, Schneider H, et al. Prematurity is related to high placental cortisol in preeclampsia. Pediatr Res. 2009;65:198-202.

13. Walsh MC, Bell EF, Kandefer S, Saha S, Carlo WA, D'angio $C T$, et al. Neonatal outcomes of moderately preterm infants compared to extremely preterm infants. Pediatr Res. 2017;82:297-304.

14. Bajaj M, Natarajan G, Shankaran S, Wyckoff M, Laptook AR, Bell EF, et al. Eunice Kennedy Shriver National Institute of Child Health and Human Development Neonatal Research Network. delivery room resuscitation and short-term outcomes in moderately preterm infants. J Pediatr. 2018;195:33-38. e2

15. Kapadia VS, Lal CV, Kakkilaya V, Heyne R, Savani RC, Wyckoff MH. Impact of the neonatal resuscitation program-recommended low oxygen strategy on outcomes of infants born preterm. J Pediatr. 2017;191:35-41.

16. Kattwinkel J, Perlman JM, Aziz K, Colby C, Fairchild K, Gallagher J, et al. Neonatal Resuscitation: 2010 American Heart Association Guidelines for cardiopulmonary resuscitation and emergency cardiovascular care. Pediatrics 2010;126: e1400-e1413.

17. Wyckoff MH, Aziz K, Escobedo M, Kapadia V, Kattwinkel J, Perlman JM, et al. Part 13:Neonatal resuscitation: 2015 American Heart Association Guidelines for cardiopulmonary resuscitation and emergency cardiovascular care. Circulation 2015;132:S543-60.

18. Sanchez AM MS, Jimenez JM, Manroe BL, Rosenfeld CR, Tyson TE. Systems approach to the evaluation of maternal and neonatal care. Proc 12th Hawaii Int'l Conf Syst Sci. Selected Papers in Medical Information Processing. 1979; III: 140151.

19. Kaiser JR, Tilford JM, Simpson PM, Salhab WA, Rosenfeld CR. Hospital survival of very-low-birth-weight neonates from 1977 to 2000. J Perinatol. 2004;24:343-50.

20. Papile LA, Burstein J, Burstein R, Koffler $H$. Incidence and evolution of subependymal and intraventricular hemorrhage: a study of infants with birth weights less than 1,500 gm. J Pediatr. 1978;92:529-34.

21. Olsen IE, Groveman SA, Lawson ML, Clark RH, Zemel BS. New intrauterine growth curves based on United States data. Pediatrics 2010;125:e214-e224.

22. McIntire DD, Bloom SL, Casey BM, Leveno KJ. Birth weight in relation to morbidity and mortality among newborn infants. N Engl J Med. 1999;340:1234-8.

23. Peduzzi P, Concato J, Kemper E, Holford TR, Feinstein AR. A simulation study of the number of events per variable in logistic regression analysis. J Clin Epidemiol. 1996;49:1373-9.

24. Amiya RM, Mlunde LB, Ota E, Swa T, Oladapo OT, Mori R. Antenatal corticosteroids for reducing adverse maternal and child outcomes in special populations of women at risk of imminent preterm birth: a systematic review and meta-analysis. PLoS One. 2016;11:e0147604.

25. Battarbee AN, Sandoval G, Grobman WA, Bailit JL, Reddy UM, Wapner RJ, et al: Eunice Kennedy Shriver National Institute of Child Health Human Development Maternal-Fetal Medicine Units (MFMU) Network. Antenatal Corticosteroids and Preterm Neonatal Morbidity and Mortality among Women with and without Diabetes in Pregnancy. Am J Perinatol. 2020 Jul:https://doi.org/10.1055/s-00401714391

26. LeVan JM, Wyckoff MH, Ahn C, Heyne R, Sánchez PJ, Chalak L, et al. Change in care among nonenrolled patients during and after a randomized trial. Pediatrics 2013;132:e960-70.

27. Smithhart W, Wyckoff MH, Kapadia V, Jaleel M, Kakkilaya V, Brown LS, et al. Delivery room continuous positive airway pressure and pneumothorax. Pediatrics 2019;144:e20190756.

28. Kakkilaya V, Jubran I, Mashruwala V, Ramon E, Simcik VN, Marshall M, et al. Quality improvement project to decrease delivery room intubations in preterm infants. Pediatrics 2019;143:e20180201. pii 
29. Gyamfi-Bannerman C, Jablonski KA, Blackwell SC, Tita ATN, Reddy UM, Jain L, et al. Eunice Kennedy Shriver National Institute of Child Health Human Development Maternal-Fetal Medicine Units Network. Evaluation of Hypoglycemia in Neonates of Women at Risk for Late Preterm Delivery: An Antenatal Late Preterm Steroids Trial Cohort Study. Am J Perinatol. 2021; May. https://doi.org/10.1055/s0041-1729561. Epub ahead of print. PMID: 34044454.

30. Reddy UM, Deshmukh U, Dude A, Harper L, Osmundson SS. Society for MaternalFetal Medicine Consult Series \#58: Use of antenatal corticosteroids for individuals at risk for late preterm delivery: Replaces SMFM Statement \#4, Implementation of the use of antenatal corticosteroids in the late preterm birth period in women at risk for preterm delivery, August 2016. Am J Obstet Gynecol. 2021;S0002-9378 (21)00859-0. https://doi.org/10.1016/j.ajog.2021.07.023. Epub ahead of print. PMID: 34363784.

31. Altman $M$, Vanpée $M$, Cnattingius $S$, Norman $M$. Neonatal morbidity in moderately preterm infants: a Swedish national population-based study. J Pediatr. 2011;158:239-44. e1

32. Valencia G, Rojas V. Association between antenatal steroids for lung maturation and hypoglycaemia in the first $48 \mathrm{~h}$ in premature infants between 26 and 34 weeks of gestational age. Pediatr Endocrinol Diabetes Metab. 2019;25:177-1182.

33. Mitchell NA, Grimbly C, Rosolowsky ET, O'Reilly M, Yaskina M, Cheung PY, et al. Incidence and risk factors for hypoglycemia during fetal-to-neonatal transition in premature infants. Front Pediatr. 2020;8:34.

\section{ACKNOWLEDGEMENTS}

We thank Dr. Bloom, Dr. Leveno, Dr. McIntire, Dr. Pérez-Fontán and Dr. Savani for participating in the discussions that eventually led to expansion of ANS to mothers with DM and GHTN on 9/21/2015.

\section{AUTHOR CONTRIBUTIONS}

HMW, LPB and CRR conceptualized and designed the study. PJB, AT and LPB collected data. LPB carried out statistical analyses. HMW and LPB drafted the initial manuscript. All authors have revised the manuscript and approved the final manuscript as submitted and agree to be accountable for all aspects of the work.

\section{COMPETING INTERESTS}

The authors declare no competing interests.

\section{ETHICAL APPROVAL}

The study was approved by the Division of Neonatal-Perinatal Medicine and Institutional Review Boards of UT Southwestern Medical Center and Parkland Health and Hospital System. The need for individual consents was waived.

\section{ADDITIONAL INFORMATION}

Correspondence and requests for materials should be addressed to Luc P. Brion.

Reprints and permission information is available at http://www.nature.com/ reprints

Publisher's note Springer Nature remains neutral with regard to jurisdictional claims in published maps and institutional affiliations. 